\title{
PENSAR EN LA INTERVENCIÓN EN CRISIS: UNA PROPUESTA DESDE INTERVENCIONES POST DESASTRES NATURALES DE CHILE Y MÉXICO
}

\section{THINKING ABOUT CRISIS INTERVENTION: AN INTERVENTION AFTER NATURAL DISASTERS OCCURRED IN CHILE AND MEXICO}

Fecha recepción: 28 de noviembre 2018 / fecha aceptación: 23 de enero 2019

\section{Gerardo Chandía Garrido, Nery Esperanza Cuevas Ocampo ${ }^{2}$ y Yanina Gutiérrez Valdés ${ }^{3}$}

\section{Cómo citar este artículo:}

Chandía, G., Cuevas, N., y Gutiérrez, Y. (2018). Pensar en la intervención en crisis: una propuesta desde intervenciones post desastres naturales de Chile y México. Revista Pensamiento y Acción Interdisciplinaria, 4(2), 60-75. http://doi.org/10.29035/pai.4.2.60

\section{Resumen}

\begin{abstract}
Las condiciones de vida en las ciudades han traído oportunidades y espacio $s$ de desarrollo, pero también han generado riesgos cotidianos. En ocasiones nos enfrentamos a eventos naturales que tienen un carácter de devastación. Pueden ser, por ejemplo: incendios, inundaciones, así como sismos, que son impredecibles y amenazantes. Estas situaciones las viven los niños/as con impotencia, se sienten desvalidos e incapaces, de ahí surge la importancia de proponer indicaciones para intervenciones psicológicas frente a eventos catastróficos. Este trabajo es fundamental para que los niños/as aprendan, en primer lugar, a preservarse a sí mismos y ayudar a otros y, en un segundo momento, generar acciones que les permitan significar estos eventos, intentando que puedan aprender sobre sus capacidades y esto devenga en una experiencia transformadora para el desarrollo de estrategias cognoscitivas, así como de recursos emocionales. El objetivo será, por tanto, evitar que la experiencia se constituya en algo traumático. Presentamos en este artículo la recuperación de dos experiencias de intervención con niños y niñas, incorporando la dimensión del cuerpo simbolizado hacia la expresión vía el arte y la palabra y considerando el eje de participación como un tema transversal en toda intervención. Se narra el trabajo con aquellos niños/as que sufrieron un gran incendio en Chile y a continuación las vivencias de los sismos en México.
\end{abstract}

Palabras Clave: Desastres Naturales; Crisis; Arte Terapia; Imagen Corporal; Intervención; Niños/as; Participación.

\footnotetext{
${ }^{1}$ Chileno, Psicólogo, Magíster Salud Mental Infanto Juvenil. Académico Dpto. de Psicología Universidad Católica de la Maule, Talca, Chile. E-mail: gchandia@ucm.cl

${ }^{2}$ Mexicana, Psicóloga, Licenciatura en Psicología, Maestra en Desarrollo Educativo Profesora, Investigadora Titular C. Ciudad de la Universidad Autónoma Metropolitana Xochimilco, Ciudad de México, México. E-mail: nerycuevas@yahoo.com.mx

${ }^{3}$ Chilena, Psicóloga, Magíster Psicología Comunitaria. Académica Dpto. de Psicología Universidad Católica de la Maule Talca, Chile. E-mail: yamaguva@gmail.com
} 


\section{Abstract}

Living conditions in cities have brought opportunities and spaces for development, but they have also generated daily risks. Sometimes we are faced with natural events that have a devastation character. They can be, for example: fires, floods, as well as earthquakes, which are unpredictable and threatening. These situations are experienced by children with impotence, they feel helpless and incapable, hence the importance of proposing indications for psychological interventions in the face of catastrophic events. This work is fundamental for children to learn, firstly, to preserve themselves and help others and, secondly, to generate actions that allow them to mean these events, trying to learn about their capacities and this becomes a transforming experience for the development of cognitive strategies, as well as emotional resources. The objective will therefore be to prevent the experience from becoming traumatic. In this article we present the recovery of two intervention experiences with children, incorporating the dimension of the body symbolized towards expression via art and words and considering the axis of participation as a transversal theme in all intervention. It narrates the work with those children who suffered a great fire in Chile and then the experiences of earthquakes in Mexico.

Keywords: Natural Disasters; Crisis; Art Therapy; Corporal Image; Intervention; Children's; Participation.

\section{Introducción}

En el mundo existen diversas situaciones de riesgo propias del cotidiano vivir donde lo que aparece como un fenómeno natural o un accidente tiene más probabilidades de volverse catástrofe si las personas viven en zonas expuestas a la pobreza y precariedad. La pobreza no es solamente la falta de bienes, sino la pauperización de los modos de vida, en cuanto a acceso a la educación, salud, servicios en general que se traducen en vulnerabilidad ante eventos (Comisión Económica para América Latina y el Caribe [CEPAL], 2017). En la región latinoamericana, a pesar de una larga historia de sismos, huracanes, incendios, inundaciones, existe la creciente necesidad de una acción más comprometida para actuar ante las crisis.

Cuando hay una situación que atender emerge ese sujeto que se compromete y desea intervenir, y anhela ayudar de manera altruista. Sin embargo, ese esfuerzo desinteresado puede ser muy costoso emocionalmente para la persona que se enfrenta a escenarios terribles, donde sus acciones pueden ser ineficaces por falta de preparación. Ese entusiasmo debe ser apoyado de una manera cuidada, tanto para interventores como para las víctimas, de otra manera acaban volviéndose víctimas todos, en cierta medida. Ante la clara posibilidad de que los eventos de riesgo se repitan, los psicólogos tienen la urgente demanda de prepararse en el campo de intervención en crisis para actuar antes y después de los eventos. El interés ha de orientarse hacia los niños, ya que aún están en proceso de desarrollo de estrategias cognoscitivas para resolver problemas, de constituir un acervo de defensas de su psiquismo y deben pasar la pubertad para asumir su 
cuerpo simbolizado. Esta mirada permite pensar en la inmadurez y vulnerabilidad de la infancia, sin embargo, puede ser pensada también como posibilidad, dependiendo de un entorno que permita potenciar sus capacidades, considerando la participación como un eje transversal.

Cada niño requiere de una comunidad que le facilite un entorno seguro donde transite de lo indefinido hacia lo verdadero, permitir que emerja la infancia como alteridad y enigma, lo que da cuenta de la necesidad de construir otro tipo de relación. La utopía está en pensar que un niño alcanza lo verdadero en el instante mismo en que aparece como alguien singular e irrepetible, como una pura diferencia, más allá de cualquier concepto, como una pura presencia irreductible a cualquier causa, condición o fundamento, como una realidad que no puede ser jamás tratada como un instrumento, como un enigma que nos mira cara a cara. La infancia es extraordinariamente compleja, recupera la historia de la familia, habla por los ancestros, pero también por sus futuros descendientes. La infancia es encarnada por un ser-ahí, eminentemente un ser histórico-social, que a través de los múltiples discursos compendia los diferentes tiempos en una suerte de malabarismo y paulatinamente logra tener un horizonte social, de modo que su mundo individual se amplía en la historia colectiva. Cada niño es potencia, es posibilidad. En dicho sentido, la educación no debiese acentuarse en la inmadurez - o lo que le falta- sino en lo que puede llegar a lograr. El destino de cada niño es necesariamente un destino individual pero también es destino colectivo. El destino individual del ser-ahí, en y con su generación, constituye en su plenitud y en su autenticidad la historicidad el ser-ahí (Zambrano, 1993).

Los niños desde pequeños van construyendo una narrativa de sí mismos y de su familia que les permite poner un cierto orden en sus emociones y apuntan a comprender lo que ocurre a su alrededor. Esta narrativa se alimenta de su experiencia, así como de las condiciones vinculares en las que vive. Cuando se encuentran ante una situación catastrófica, sus mecanismos de defensa pueden ser insuficientes y amplificar un estado temprano de indefensión y vulnerabilidad. En este punto es donde es posible hacer la diferencia y trazar un camino del héroe, a la manera de Campbell, donde el protagonista pasa por una serie de dificultades y en su recorrido sale fortalecido, donde podemos fomentar una nueva narrativa hacia su desarrollo pleno (Campbell, 1949).

¿Qué tal proponerle a un niño algo así? Al producirse un evento inesperado la situación habitual se modifica y aparece la incertidumbre, surgen los temores, la ansiedad y posteriormente la movilización para afrontar dicha condición emergente. ¿Qué ocurre al concebir al niño como un sujeto activo en su conocimiento, creativo en su actuar y como posible agente de cambio? Démosle la palabra al niño (Dolto, 1985). 


\section{Marco referencial}

\section{Catástrofes de origen natural}

Por catástrofes, en términos generales, se comprende a un sinnúmero de eventos no esperados de impacto - directo o indirecto- en la vida de las personas. Estos eventos convocan a que muchas disciplinas intenten dar respuesta a estos fenómenos (ya sea desde la psicología, sociología, geología, física, geografía, urbanismo, etc.) pero en ocasiones muy poco conectadas entre ellas, lo que conlleva a respuesta fragmentadas frente al desastre (Capacci y Mangano, 2015).

Dentro de las catástrofes de origen natural tenemos los terremotos, erupciones, las inundaciones, deslizamientos, los huracanes, olas de calor y frío intensos, las tormentas, plagas, infecciones causadas por bacterias y virus, plantas venenosas, la fiebre del heno, entre otras. De origen seminatural encontramos el smog, la desertificación, avalanchas, el calentamiento global, etc. Y por último, de origen antrópicos tenemos la contaminación, los desastres industriales y las guerras (Capacci y Mangano, 2015). En el presente trabajo nos centraremos en dos eventos de origen natural: terremotos e incendios.

\section{Consecuencias esperadas de una catástrofe}

Al enfrentar un evento de esta naturaleza nuestra respuesta es la angustia, la huida y el temor, que finalmente apuntan a la fragmentación, descrita por Melanie Klein como forma básica y temprana de manejo de la angustia, como estrategia ante aquello que rebasa nuestras posibilidades de respuesta (Guerra, Plaza, y Vargas, 2018; Organización Panamericana de la Salud [OPS], 2006). Esta angustia que vivimos es superior a nuestro dique defensivo y nuestros mecanismos de defensa se orientan a lo básico, la escisión. Posteriormente, como sujetos complejos, los niños tendrán un movimiento de búsqueda de sentidos, los procesos de producción de la subjetividad en un intento de construir configuraciones, orientadoras en su psique.

Las consecuencias más frecuentes, ante las situaciones de riesgo, son las que evidencian la tendencia a escindir, es decir, las crisis de ansiedad, la dificultad para ordenar tiempos y espacios, el llanto, el temor, la falta de atención y concentración, y los procesos de regresión, entre otros. La calidad del pensamiento depende de manera fundante del eje espacio-temporal, así como la posibilidad de planear y anticipar. Cuando esto se vuelve impredecible nuestra capacidad de pensar se ve afectada, surgen pensamientos circulares, repetitivos, sin progresión, lo que dificulta el uso de mecanismos de defensa más elaborados. La merma en capacidades puede inscribirse en el cuerpo manifestándose con torpeza, fallas en coordinación y equilibrio. Las personas son seres simbólicos, la materia del 
pensar es lo representado tanto en nuestra imagen corporal como en la palabra, de otra manera lo que enfrentamos es inasible y potencialmente perturbador, y requiere significar para afrontar el riesgo y actuar hacia la preservación.

A nivel comunitario, los entornos sociales ven afectadas sus capacidades de afrontamiento lo que influye directamente en su bienestar, impactando además el tejido social por desestructuración, lo que impide un funcionando con normalidad (Beristain, 2000). En este contexto, se señalan 3 aspectos que influyen en cómo un evento catastrófico influye a nivel comunitario: (1) la naturaleza misma del evento, (2) las características de personalidad de los afectados y vulnerabilidades previas y (3) las circunstancias de ocurrencia del evento (Paéz, Fernández, y Beristain, 2001). Por otra parte, el fenómeno de desplazamiento del territorio post catástrofe igualmente genera en los sujetos situaciones complejas. Entre ellas, las dificultades para adaptarse a los nuevos territorios y comunidades, el alejamiento de las redes de apoyo y el abandono del estilo de vida previo (Espinoza, 2014; Lillo, 2013; Marchant, 2010). Las investigaciones señalan que incluso cuando los procesos migratorios son realizados de manera favorable, igualmente implica una situación de crisis con los consiguientes efectos psicosociales (Bar de Jones, 2011; Briones, 2010; Oficina Nacional de Emergencia del Ministerio del Interior [ONEMI], 2006).

La intervención psicológica apunta a mover el caos hacia la organización donde la tarea esencial es fomentar la coherencia psíquica y la congruencia con el entorno, y para ello son fundamentales la contención afectivo-cognoscitiva y el proporcionar un ambiente con interacciones estables. Es decir, ante la fragmentación y dispersión la demanda es hacia la continuidad y configuración armoniosa del entorno. Esto ofrecerá al niño la bondad de un referente que le permita reintegrar sus recursos y orientarse en sus acciones (Oaklander, 2012).

\section{¿Cuándo intervenir?}

En zonas de riesgo es importante intervenir, idealmente, antes de las crisis, desde que los niños están en niveles preescolares. Al respecto, hay países donde la educación a la ciudadanía, incluidos niños y niñas, incorpora una preparación para reaccionar en situaciones de desastre. Esto requiere, el apoyo de una institucionalidad que acompañe estos procesos. Entre estos países, Japón tiene una larga tradición en la identificación y manejo de riesgos de desastres (Roque, Criollo, Recino, Gafas, y Barahona, 2016). En otras ocasiones, los profesionales apoyan en el desastre una vez ocurrido. En ambos casos, el proceder se puede orientar hacia la realización de intervenciones que propicien una imagen corporal integrada, autoestima, respeto y, especialmente, acciones tendientes a formar en los niños lazos de solidaridad y apoyo mutuo. Con esta base podemos trabajar 
la gestión de riesgos, preparar a los niños para actuar con autocuidado y cooperando con los demás. Un eje fundante es la realización de la intervención en un ambiente de respeto, es decir, con la profunda valoración de la cabal alteridad de la infancia, sin pretender caer en extremos como la sobreprotección o el exceso de normas sino con una contención afectiva e interés genuino (Oaklander, 2012).

Es esencial que el ambiente de intervención sea tranquilo y que exista una prima de placer en el ejercicio, es decir la presencia del humor y el juego pueden crear un ambiente propicio para la elaboración de la angustia asociada. Será necesario transitar en un programa que parta de actividades de orden corporal hacia el grafismo y posteriormente la palabra (Dolto, 1986). El niño necesita sentirse seguro para poder llegar a nombrar, por lo cual no debemos apresurarnos y solicitar que hablen, los procesos simbolizadores son procesos de tiempo y espacio. Para ello, lo primero que requiere atención son los profesionales, para que estén preparados para atender la crisis con autocontrol y tranquilidad.

\section{Objetivos de la intervención}

Considerando lo señalado por la Organización de las Naciones Unidas (ONU) en torno al abordaje de las situaciones de desastres naturales, se han establecido distintas estrategias asociadas a la gestión del riesgo y la prevención de desastres (Campos, 2000; Muñoz, 2010). En este sentido, el objetivo de la intervención preventiva es lograr que los niños puedan vivir el riesgo habiendo desarrollado mejores recursos emocionales y estrategias cognoscitivas, y en la intervención posterior al evento ofrecer al niño una convocatoria relacional que sostenga su integridad psíquica, ofreciéndole un programa de actividades que le permita acceder a significados y generar sentidos de sí, y del evento en cuestión, para que actúe cuidándose a sí mismo y con mutualidad, incluyendo el espacio comunitario como un sostenedor de las situaciones vividas.

\section{Hacia una crisis productiva, generando una oportunidad}

Las formas de crianza actuales se enfrentan a retos ante lo incalculable que puede ser de orden de la naturaleza misma, así como de orden social que fragilizan las nuevas subjetividades. Consideramos urgente atender a los adultos a cargo de los niños para que incorporen en sus decisiones cotidianas el horizonte de gestión del riesgo (Giddens, 2000).

Cuando el riesgo se concreta en un evento, es decir en una crisis, la intervención puede ser este punto de decisión que permite que la situación se convierta en una experiencia transformadora, al propiciar el paso de la incertidumbre y vulnerabilidad hacia la capacidad de hacer algo, de aportar en la medida de las 
posibilidades. Si el riesgo es afrontable es posible cambiar la narrativa de una situación que vulnera a una situación a superar, una situación que demanda la propia solidaridad.

\section{Niños como agentes del cambio, no víctimas}

La exposición a situaciones que se caracterizan por generar incertidumbre demanda desarrollar programas sistemáticos, pertinentes, continuos y de un compromiso profundo con los niños. Estos aspectos son fundamentales para refrendar la constancia afectiva y de presencia, ofrecer un espacio de lo anticipable que se constituya en un espacio de control, ya que el niño necesita referenciarse en lo que permanece para pasar de la fragmentación a la integración. Lo siguiente es reconocer el temor colectivo, para transitar de lo individual a lo solidario y la convocatoria a convertirse en agente causal que permita al niño dirigir su angustia hacia una meta.

Uno de los recursos ante los desastres es la resiliencia que puede ser pensada como la manera de responder a los riesgos, a lo largo del tiempo, con capacidad de afrontamiento y superación. Originalmente se le refería como la característica de ciertos materiales que después de modificar su forma, cuando se les aplica fuerza, pueden recuperar su forma original. Los sujetos somos más complejos por lo cual podemos pensar a la resiliencia con cierta libertad. Un niño es resiliente si tiene la fuerza para resistir situaciones de dificultad, carencia o riesgo y sale adelante. Sin embargo, ese ejercicio de afrontamiento genera cambios en el niño, donde no regresará a su condición previa y su subjetividad se modifica. Las personas resilientes se caracterizan por ser flexibles y tienden a un pensamiento más complejo e interpretan la realidad de una manera menos impulsiva (American Psychological Association [APA], 2015). Los psicólogos podemos crear condiciones para lograr la resiliencia en los niños a través de: a) promover que el niño conozca sus habilidades y confíe en ellas, b) trabajar en la significación de sus emociones, primero es importante que las reconozca, las asocie a situaciones específicas y logre ser empático con los otros, c) practicar el control de impulsos, procurando detenerse antes de actuar, d) socializar los temores a través del diálogo, e) desarrollar una narrativa que permita significar los desastres, y f) proponer acciones a tomar ante el evento.

¿Pero cómo lograrlo? Acompañando al niño en actividades grupales, ordenadas, utilizando vías simbólicas como el movimiento en relación a tiempo y espacio, movimiento asociado a narrativa, dibujo, cuento, juego, música, entre otros. Las líneas que guían son las del psicodesarrollo, trabajando de lo simple a lo complejo, de lo general a lo particular y de lo global a lo específico. Se involucran propuestas de tareas recuperando los puntos claves del desarrollo donde se 
anudan lo corporal, lo cognoscitivo y afectivo. Por ejemplo, al moverse de acuerdo a la narrativa de un cuento debemos contemplar la complejidad de la situación, del lenguaje, de lo témporo-espacial y dominios motrices para proponerle la actividad al niño, donde ese "barco de piratas en una tormenta" permita escenificar las situaciones de riesgo, lo inesperado y lo controlable y la importancia de la cooperación mutua.

\section{Participación Infantil}

La Convención de los Derechos del Niño (1989) realizada a través de la Carta Magna de la Infancia (Alfageme, Cantos, y Martínez, 2003), constituye el reconocimiento de los niños, niñas y adolescentes como sujetos de derechos y personas activas en su propio proceso de desarrollo, lo que constituyó un cambio del cómo visualizar está etapa del ciclo vital. Dentro de los derechos consagrados aparece el derecho a participar (definido como el derecho a expresar su opinión y que ésta sea tenida en cuenta), el derecho a la libertad de expresión, el derecho a la libertad de pensamiento y el derecho a ser informado (Castro, Ezquerra, y Argos, 2016).

No obstante, la realidad nos indica que este cambio de paradigma ha generado constantes resistencias a nivel social (Apud, 2001) encontrándonos con una mirada adultocentrista que dificulta aceptar la participación infantil (Contreras y Pérez, 2011). Tal como señala Larsen (2011), la participación lleva consigo el involucramiento de distintos enfoques entre ellos el de ciudadanía, la teoría relacional, la perspectiva ética y los derechos humanos (Backe-Hansen, 2009; Lister, Smith, Middleton, y Cox, 2003; Rebellato, 1995).

Hoy la investigación ha demostrado avances que dan cuenta de este nivel de participación, encontrándonos con estudios que promueven una visión de los niños/as como sujetos activos, convirtiendo los adultos en co-intérpretes de la realidad que experimentan (Clark, 2005; Dockett y Perry, 2007; Harcourt, 2011; Lay-Lisboa y Montañés, 2018; Mannion, 2010).

Por otro lado, los organismos internacionales han llegado a acuerdos respecto de procedimientos frente a emergencias donde se priorice la atención a niños y niñas. El año 2011 se elaboró una Carta de La Niñez para la Reducción de Riesgo de Desastres, para lo cual fueron consultados alrededor de 600 niñas y niños de diferentes países en África, Asia y Latinoamérica. En esta consulta niños y niñas plantearon prioridades frente a los desastres, una de las cuales es el derecho a participar y tener acceso a la información que necesitan (Organización de la Naciones Unidas, 2015). 
La escuela constituye el lugar preciso para que niños/as y familias reciban servicios de atención psicosocial. Es un punto de encuentro que permite sostener acciones y contener a las comunidades en situaciones de crisis (Programa de Apoyo a la Convivencia Escolar, 2014). Por lo mismo, es considerada una fuente de resiliencia comunitaria.

El modelo adoptado es el propuesto por Hart (1992) en su escala de participación infantil que define 8 niveles, dentro de los cuales los 3 primeros indican una no participación (manipulación, decoración, participación simbólica) y los 5 niveles restantes implican una nivel de participación que va aumentando a medida que sube en la escala (información, consulta, iniciado por adultos, iniciado por niños/as, compartido con personas adultas).

\section{Dos propuestas de Intervención}

\section{Mega incendio forestal en Chile el año 2017}

Chile se caracteriza por la presencia de desastres naturales de manera periódica, entre ellos terremotos, tsunamis, erupciones volcánicas e incendios forestales (Espinoza, Espinoza, y Fuentes, 2015). En este contexto durante el verano del año 2017 se produce un mega incendio en la localidad de Santa Olga, Constitución, Región del Maule, Chile, destruyendo un total de 160 mil hectáreas, lo que significó la destrucción total de dicha localidad, dejando un total de 5.000 personas sin vivienda. Dentro de las orientaciones entregadas por el Estado de Chile se cuenta el traslado de la población a la localidad de Constitución, reubicándose además el colegio de Enrique Mac Iver de Santa Olga. En ese marco desde la Escuela de Psicología de la Universidad Católica del Maule, en convenio con la Fundación de Superación de la Pobreza de la Región del Maule, se diseñó un plan de trabajo que permitiera contribuir en el proceso de acompañamiento de los niños/as afectados. La primera estrategia fue definir junto al equipo técnico del colegio el grupo de niños/as menos intervenido, considerando que post incendio surgió mucha ayuda, decidiéndose trabajar con los $5^{\circ}$ básicos de educación escolar. Es así como surge el proyecto "Creando Juntos" desarrollado en el año 2017 con 12 estudiantes de pregrado de la Carrera de Psicología, quienes recibieron capacitación en el uso de materiales artísticos y en el acercamiento hacia personas que experimentan una catástrofe. Es así como durante el año 2017 se implementó un taller de expresión artística con niños y niñas de $5^{\circ}$ año básico (dos cursos, 60 niños/as), con una totalidad de 10 sesiones ocupando como eje de intervención los recursos artísticos y la participación infantil. El taller finalizó con una feria de creatividad, donde se presentaron las producciones artísticas hechas por los estudiantes a la comunidad escolar. Durante el año 2018 se decidió continuar con el trabajo con los mismos estudiantes (actualmente en $6^{\circ}$ básico), contando nuevamente con la colaboración de estudiantes de Psicología, y además de profesionales de la Fundación 
de Superación de la Pobreza, quienes además aportaron con ayuda financiera y técnica. El eje del trabajo fue la preparación al nuevo escenario vinculado al proceso de reconstrucción de la comunidad de Santa Olga, lo que implicó reconocer que algunos niños/as ya habían regresado a sus nuevas viviendas y otros se mantenían en Constitución, estimándose la entrega del nuevo establecimiento educacional para el año 2019, por tanto, el objetivo se dirigió a que los niños/as pudieran expresar y narrar el proceso de reinserción al territorio reconstruido, post desastre natural, a través de técnicas narrativas. Las estrategias a desarrollar son sesiones de trabajo semanal, realizándose un total de 10 sesiones. Actualmente el proyecto se encuentra en fase de evaluación y en la confección de un libro que sistematizará la experiencia rescatada desde los niños/as.

Dentro de los aprendizajes obtenidos durante estos dos años de trabajo se visualiza la necesidad de reconocer las experiencias de los niños frente a situaciones de desastres, dejando de lado miradas que tienden a minimizar las situaciones bajo el lema que la "vida debe continuar", como también el abordaje de situaciones dolorosas y procesos individuales que tienen tiempos distintos y variadas formas de expresar el malestar. Se ha enfatizado el trabajo en pequeños grupos, de tal forma que esta experiencia colectiva permita el intercambio, normalización, emergencia de recursos internos, y otros elementos de subjetivación grupal (González Rey, 2002). Desde ahí se plantea el cómo niños y niñas aportarán en el proceso de retorno al lugar, desde posiciones de creación y recreación cultural (Gutiérrez, Chandía, y Vergara, s/f). Niños y niñas participantes elaboran pequeños relatos ficcionados que les permiten integrar, por una parte, sus recursos personales y por otra, sus temores y fantasías respecto de nuevos sucesos de desastre. Durante el año 2017 la temática del incendio no aparece como un factor relevante en lo reportado por los niños, sin embargo durante la intervención del año 2018, la temática reaparece vinculada al proceso de retorno y el surgimiento de temores asociados a la experiencia traumática. Pretendemos generar un tercer año de trabajo que implique efectivamente el traslado del liceo al territorio.

\section{Los sismos de Ciudad de México en el año 2017}

Ciudad de México se ha caracterizado a lo largo de su historia, por ser escenario de múltiples sismos, sin embargo, la intervención psicológica ha sido insuficiente. En especial si sabemos que seguirán presentándose estos eventos, es fundamental pensar en formar a los futuros psicólogos en el área de intervención en crisis, así como en el diseño de protocolos de acción.

Durante el mes de septiembre del año 2017 se vivieron varios sismos de diferentes magnitudes, que impactaron tanto los inmuebles como las certezas y 
modos de vida. Los niños presentaron signos de afectación como ansiedad, llanto, dificultades de concentración, incapacidad de adquirir nuevos conocimientos, hiperreactividad, miedo, entre otros. Para atender esta situación se diseñó un programa de intervención con niños de tres a cinco años de edad en espacios escolares.

Se trabajó con alumnos de la Licenciatura en Psicología de la Universidad Autónoma Metropolitana Xochimilco, ubicada en la zona sur de la Ciudad de México. Se realizaron actividades de relajación, técnicas corporales, dibujo y grupos de discusión con los alumnos para que ellos pudieran manejar su propia angustia. Estas acciones se llevaron a cabo en una escuela pública de educación básica, con dos grupos de quince y veinte niños. La elección del espacio escolar se basó en la posibilidad de sistematizar la intervención al contar con una continuidad de asistencia de los grupos, espacios que conocen considerando que el sismo de mayor intensidad ocurrió en ese lugar. Se consideró que era necesario resignificar el espacio escolar como un lugar de prevención y participación donde el niño puede actuar.

Se realizaron doce sesiones con los niños de etapa preescolar, con la conducción de dos alumnos por grupo, que acompañaron al niño en actividades grupales, ordenadas, utilizando vías simbólicas como el movimiento en relación a tiempo y espacio, movimiento asociado a narrativa, dibujo, cuento, juego, música, entre otros, organizados en relación a un recorrido de actividades que parten de lo fundamental, la apropiación simbólica del cuerpo como un recurso de continuidad e identidad ante el cambio. Se inicio con el registro de un cuerpo completo, a través del movimiento, hacia la complejidad que llevará a la palabra.

Los puntos abordados, de manera secuencial en el programa son cuerpo total, cuerpo diferenciado, cuerpo de tiempos y espacios, cuerpo y palabra, cuento, dibujo, elaboración de máscaras de sus temores, dramatización, socialización de sus temores y participación en apoyo a otros.

Los caminos del psicodesarrollo son paradójicamente compartidos y singulares. Por lo cual crear entornos que permitan al niño volver a recorrer los senderos en sus tiempos y su manera le permite restaurar y consolidar los procesos (Oaklander, 2012). A través de su comportamiento los niños están realizando preguntas a las personas que les rodean, en este recorrido cada niño encontrará respuestas diferentes porque sus preguntas son diferentes. El sismo los llevó a revisitar rasgos de etapas previas, pero no todos regresan a los mismos ni de la misma manera, por eso se acompañó, con continuidad y contención, en una revisión desde sus procesos actuales para que puedan readquirir los dominios yoicos logrados y desarrollen recursos para ser resilientes. 
Después de doce sesiones observamos que los niños tuvieron mejor control de impulsos y manejo de la ansiedad, la mayoría logró mayor concentración y aprendizaje. Con base en los resultados, se concluye que si el bien el programa ayudó a restaurar algunos de los logros del desarrollo lo ideal sería prepararse. Por lo cual, estas actividades deberían ser parte de los programas escolares, si los niños practican qué deben hacer en caso de sismo en un ambiente cordial estamos promoviendo la simbolización y la inscripción de las acciones en el cuerpo, así como la posibilidad de ayudar a otros convoca procesos de pensamiento hacia la madurez y solidaridad.

\section{Conclusiones}

A partir de las experiencias sucintamente relatadas en Chile y México luego de eventos naturales tales como sismos e incendios, donde se ha trabajado con niños y niñas en contextos escolares, se pueden concluir algunos elementos comunes. En primer lugar, como se dijo anteriormente, en estos procesos se ha hecho uso del espacio escolar como punto de encuentro y aprendizaje tanto individual como colectivo. Los establecimientos educacionales constituyen lugares privilegiados donde se pueden promover entrenamientos tanto para niños-niñas como para las personas adultas que se relacionan con estos sistemas. En el caso de Santa Olga, a raíz del incendio producido, toda la localidad fue arrasada, lo cual implicó que se produjera una diáspora de sus habitantes abandonando este territorio. Acá entonces, se puede ver que el espacio de representación del lugar lo mantuvo el establecimiento educacional que fue trasladado a la ciudad más cercana. Desde esta perspectiva es necesario preguntarse ¿dónde se hace una intervención centrada en el territorio si este resulta devastado y su población desagregada? La respuesta natural es el lugar que convoca y reúne a la mayor cantidad de personas que son parte de esta comunidad fragmentada temporalmente como consecuencia del fuego. La escuela constituye un espacio que contiene, crea y recrea parte de la identidad local (Redón, 2011). El segundo elemento en común de ambas intervenciones tiene que ver con la aproximación desde el reconocimiento de las sensibilidades y afectos involucrados en las situaciones de crisis. Estas dimensiones, en ocasiones quedan invisibilizadas por las diversas urgencias que surgen en los contextos removidos por eventos ocurridos en la naturaleza que alcanzan la categoría de desastre. El trabajo con niños y niñas que han sido participantes de estos eventos, desde aproximaciones respetuosas con sus tiempos y funcionamiento psicológico, resulta indispensable para llegar a acercamientos centrados en el protagonismo y la participación infantil. El apoyo mutuo, considerado acá como el sostener intercambios grupales a través del uso de recursos artísticos, la vinculación con el movimiento y espacio, el ficcionar relatos, personajes y narraciones, la creación de objetos tridimensionales, entre otras técnicas, facilita el procesamiento de experiencias y/o sensaciones confusas, difíciles de verbalizar. Ambas experiencias relatadas dan cuenta de elementos grupales inte- 
resantes que apuntan a generar lazos entre niños y niñas, tejiéndose pequeñas redes de apoyo y soporte. Por último, ambas intervenciones se sostienen con el trabajo de estudiantes en formación que reciben entrenamiento previo para contener la propia emocionalidad y la de otros, en este caso, niños y niñas. Este último punto permite proyectar la necesidad de incorporar en los procesos de formación terciaria, espacios de entrenamiento y aprendizaje sobre intervención en crisis en situaciones de desastre. Gestionar el riesgo de manera clara y como parte de una planificación previa que se traduce, por ejemplo, en protocolos de acción para adultos/as, jóvenes, niños y niñas, personas mayores, posibilita un manejo más efectivo y puede disminuir el impacto de las crisis generadas por eventos catastróficos o de desastres.

\section{Referencias}

Alfagente, E., Cantos, R., y Martínez, M. (2003). De la participación al protagonismo infantil. Propuestas para la acción. Madrid: Plataforma de Organizaciones de Infancia (POI).

American Psychological Association APA. (2015). Guía de resiliencia para padres y maestros. Recuperado de: https://www.apa.org/centrodeapoyo/guia.aspx

Apud, A. (2001). Partcipación infantl. Enrédate con UNICEF. Formación del profesorado.

Backe-Hansen, E. (2009). Darns medbestemmelse sett med barns øyne. En Hjermann, R. \& Haanes, K. (red.), Barn (52-69). Oslo: Universitetsforlaget.

Bar de Jones, G. (2011). Panel Quiebres Vitales: la migración como quiebre vital. En II Congreso Argentno de Psicoanálisis de Familia y Pareja. Teoría y Clínica de los Vínculos.

Beristain, C. (2000). Apoyo psicosocial en catástrofes colectivas: de la prevención a la construcción. Caracas. Asociación Venezolana de Psicología Social.

Briones, F. (2010). Inundados, reubicados y olvidados. Traslado del riesg de desastres en Motozintal, Chiapas. Revista de Ingeniería Universidad de Los Andes, 31, 132-144.

Campbell, J. (1949). El héroe de las mil caras. Psicoanálisis del mito. Madrid, España: Fondo de Cultura Económica de España.

Campos, A. (2000). Educación y prevención de desastres. Fondo de las Naciones Unidas para la infancia. Facultad Latinoamericana de Ciencias Sociales. Red de Estudios Sociales en Prevención de desastres en América Latina. 
Capacci, A., y Mangano, S. (2015). Las catástrofes naturales. Cuadernos de Geografa: Revista Colombiana de Geografía, 24(2), 35-51.

Castro, A., Ezquerra, P., y Argos, J. (2016). Procesos de escucha y partcipación de los niños en el marco de la educación infantil: una revisión de la investgación. Educación XXI, 19(2), 105-126.

Comisión Económica para América Latna y el Caribe CEPAL. (2017). La consecuencia poco natural de los desastres naturales en Revista Desafios. Boletín de infancia y adolescencia. Niñez y desastres en América Latina. No. 20, mayo 2017. Recuperado de: https://repositorio.cepal.org/bitstream/ handle/11362/41842/2/articulo_consecuencia.pdf

Clark, A. (2005). Ways of seeing: using the Mosaic approach to listen to Young children's perspective. En A., Clark, T., Kjorholt, y P., Moss (Eds), Beyond listening: children's perspectives on early childhood services. Bristol: Policy Press.

Contreras, C., y Pérez, A. (2011). Partcipación invisible: niñez y práctcas partcipatvas emergentes. Revista Latinoamericana de Ciencias Sociales, Niñez y Juventud, 2(9), 811-825.

Dockett, S., y Perry, B. (2007). Transitions to school: perceptions, expectations, experiences. Sydney: UNSW Press.

Dolto, F. (1985). La causa de los niños. Barcelona: Paidós.

Dolto, F. (1986). La imagen inconsciente del cuerpo. Barcelona: Paidós.

Espinoza, A. (2014). Aproximaciones a la comprensión de los efectos traumáticos del desplazamiento forzado producto de la erupción del Volcán Chaitén en la población retornada. En P. Cabrera (Comp.) Construcciones clínica de lo traumático y figurabilidad, Santiago, Chile: Colección Praxis Psicológica, FACSO.

Espinoza, A., Espinoza, C., y Fuentes, A. (2014). Retornando a Chaitén: Diagnóstco partcipatvo de una comunidad educatva desplazada por un desastre socionatural. Magallania, 43(3), 65-76.

Giddens, A. (2000). Un mundo desbocado. Los efectos de la globalización en nuestras vidas. Madrid: Taurus.

González Rey, F. (2002). Sujeto y subjetividad. Una aproximación histórico-cultural. México: Internacional Thomson Editores. 
Guerra, C., Plaza, H., y Vargas, J. (2018). Estrés postraumático en adolescentes expuestos a un mega incendio: asociaciones con factores cognitivos y emocionales. Psicoperspectivas, 17(2), 1-12.

Gutiérrez, Y., Chandía, G., y Vergara, F. (s/f). Sistematización de la intervención en Santa Olga: rehabitar las relaciones y los espacios. Manuscrito en preparación.

Harcourt, D. (2011). An encounter with children: seeking meaning and understanding about childhood. European Early Childhood Education Research Journal, 19(3), 331-343.

Hart, R. (1992). Children's participation. From tokenism to citizenship. Florence: UNICEF.

Lay-Lisboa, S., y Montañes, M. (2018). De la participación adultocéntrica a la disidente: la otra participación infantil. Psicoperspectivas, 17 (2), 1-12.

Larsen, E. (2011). Help or formality? Children's experiences of participation in home-based child welfare cases: a Norwegian example. Nordic Social Work Research, 1(1), 43-60.

Lillo, J. (2013). A 40 días de cumplirse la meta: en una semana 410 mil alumnos ya se incorporaron a clases. Ministerio de Educación.

Lister, R., Smith, N., Middleton, S., y Cox, L. (2003). Young people talk about citizenship: Empirical perspectives on theoretical and political debates. Citizenship Studies, 7(2), 235-253.

Mannion, G. (2010). After participation, the socio-spatial perfomance of

intergenerational becoming. En B. Percy-Smith y N. Thomas (Eds), A Handbook of children and young people's participation: perspectives from theory and practice. London: Routledge.

Marchant, J. (2010). Lágrimas de ceniza. Estudio cualitativo sobre la experiencia de desplazamiento de los habitantes de Chaitén, asentados en las ciudades de la Isla de Chiloé y Puerto Montt. Oficina Nacional de Emergencia.

Muñoz, V. (2010). El derecho a la educación en situaciones de emergencia. Revista Latinoamericana de Inclusión Educativa, 4(2), 59-77.

Oaklander, V. (2012). El tesoro escondido. Buenos Aires: Cuatro Vientos.

Oficina Nacional de Emergencia del Ministerio del Interior. (2006). Intervención psicosocial en situaciones de emergencia y desastre: guía para el primer apoyo psicológico. Santiago: Gobierno de Chile. 
Organización de las Naciones Unidas. (2015). Marco de Sendai para la Reducción del Riesgo de Desastres 2015-2030. Ginebra: UNISDR.

Organización Panamericana de la Salud. (2006). Guía práctica de salud mental en situaciones de desastres. Washington, D.C.: Organización Mundial de la Salud.

Páez, D., Fernández, I., y Beristain, C. (2001). Catástrofes, traumas y conductas colectivas: procesos y efectos culturales. En San Juan, C. (Ed.) Catástrofes y ayuda en emergencia: estrategias de evaluación, prevención y tratamiento. Barcelona: Icaria.

Programa de Apoyo a la Convivencia Escolar (2014). Comunidades Escolares en Situaciones de Emergencia y Desastres. Orientaciones para el Apoyo Psicosocial. Escuela de Psicología, Pontificia Universidad Católica de Valparaíso.

Rebellato, J. (1995). La encrucijada de la ética. Neoliberalismo, conflicto nortesur. Montevideo: Editorial Nordan.

Redón,S.(2011). Escuela eidentidad:undesafío docente parala cohesión social.Polis (Santiago), 10(30), 447-476. doi:10.4067/S0718-65682011000300021

Roque, Y., Criollo, A., Recino, U., Gafas, C., y Barahona, D. (2016). La educación continuada en el proceso de reducción de desastres: experiencias cubanas. Política y cultura, 45, 11-30.

Zambrano, M. (1993). Filosofía y poesía. Madrid: Fondo de Cultura Económica.

Dirección de correspondencia:

Gerardo Chandía Garrido

Psicólogo. Magíster en Salud Mental Infanto Juvenil.

Académico del Departamento de Psicología de la Universidad Católica del Maule, Talca, Chile. Contacto: gchandia@ucm.cl 